Pacific Journal of Mathematic 


\title{
SPECTRA, TENSOR PRODUCTS, AND LINEAR OPERATOR EQUATIONS
}

\author{
M. R. Embry AND M. Rosenblum
}

Suppose $\mathfrak{X}_{1}$ and $\mathfrak{X}_{2}$ are complex Banach spaces with $u_{0}, \cdots$, $u_{n}$ in $\mathscr{L}\left(\mathfrak{X}_{1}\right), v \in \mathscr{L}\left(\mathfrak{X}_{2}\right)$, and suppose $\otimes$ is a uniform crossnorm. The spectra of the operators $\sum_{j=0}^{n} u_{j} \otimes v^{j}$ on $\mathfrak{X}_{1} \otimes \mathfrak{X}_{2}$ and $R: x \rightarrow$ $\sum_{j=0}^{n} u_{j} x v^{j}, x \in \mathscr{L}\left(\mathfrak{X}_{2}, \mathfrak{X}_{1}\right)$, are studied in the context of a general theory. Explicit representations are set down for the resolvents of these and more general operators.

0. Introduction. A classical result of Stephanos [9, p. 83] can be phrased as follows:

Suppose $u$ and $v$ are complex $n \times n$ matrices and $p_{0}, \cdots, p_{m}$ are complex polynomials. Let $\otimes$ denote tensor product, and $\sigma$ spectrum. Then

$$
\sigma\left(\sum_{j=0}^{m} p_{j}(u) \otimes v^{j}\right)=\bigcup\left\{\sigma\left(\sum_{j=0}^{m} p_{j}(u) z^{j}\right): z \in \sigma(v)\right\} .
$$

In 1966 Datuasvili [3] gave the following generalization of Stephanos' result. Let $u_{0}, \cdots, u_{m}$ and $v$ be complex $n \times n$ matrices. Then

$$
\sigma\left(\sum_{j=0}^{m} u_{j} \otimes v^{j}\right)=\bigcup\left\{\sigma\left(\sum_{j=0}^{m} u_{j} z^{j}\right): z \in \sigma(v)\right\} .
$$

Stephanos' theorem can be interpreted as a result on linear operator equations. It implies that the operator $T$ on $n \times n$ matrices defined by $T x=\sum_{j=0}^{m} p_{j}(u) x v^{j}$ has

$$
\sigma(T)=\bigcup\left\{\sigma\left(\sum_{j=0}^{m} p_{j}(u) z^{j}\right): z \in \sigma(v)\right\} .
$$

Similarly Datuasvili's result yields that the operator $R$ defined by $R x=\sum_{j=0}^{m} u_{j} x v^{j}$ has

$$
\sigma(R)=\mathbf{U}\left\{\sigma\left(\sum_{j=0}^{m} u_{j} z^{j}\right): z \in \sigma(v)\right\} .
$$

Lumer and Rosenblum [8] proved that (3) holds if $u, v \in \mathscr{L}(\mathfrak{X})$, where $\mathfrak{X}$ is a complex Banach space and $T$ is considered as an operator on $\mathscr{L}(\mathfrak{X})$ to $\mathscr{L}(\mathfrak{X})$. R. E. Harte [6] has recently shown that (4) holds if $u_{0}, \cdots, u_{m}$ and $v$ are in $\mathscr{L}(\mathscr{S})$, where $\mathscr{S}$ is a complex Hilbert space.

Brown and Pearcy [1] proved that $\sigma(u \otimes v)=\sigma(u) \sigma(v)$ in case $u, v \in \mathscr{L}(\mathfrak{S})$ and $u \otimes v$ acts on the Hilbert space $\mathfrak{S} \otimes \mathfrak{S}$. This was 
generalized by Schechter [12] and Dash and Schechter [2]. It was further generalized by Harte in [6].

In this paper we shall set down explicit representations for the resolvent of each of

(i) $\sum_{j=0}^{n} u_{j} \otimes v^{j}$,

where $u_{0}, \cdots, u_{n} \in \mathscr{L}\left(\mathfrak{X}_{1}\right), v \in \mathscr{L}\left(\mathfrak{X}_{2}\right)$, and $\otimes$ is any uniform crossnorm, and

(ii) $\quad R: x \rightarrow \sum_{j=0}^{n} u_{j} x v^{j}, x \in \mathscr{L}\left(\mathfrak{X}_{2}, \mathfrak{X}_{1}\right)$, where $u_{0}, \cdots, u_{n} \in \mathscr{L}\left(\mathfrak{X}_{1}\right)$ and $v \in \mathscr{L}\left(\mathfrak{X}_{2}\right)$. For a survey of explicit solutions of linear matrix equations, see [7].

The theory for the representations is presented in $\S 1$. In $\S 2$ we prove a spectral mapping theorem that subsumes conclusions such as those of (2) and (4) in one unified theory. In $\S 3$ we give some applications.

The notation and terminology used in the paper are as follows. $\mathfrak{U}$ will denote a complex Banach algebra with identity 1 or $I$. If $\alpha \in \mathfrak{N}, \sigma(\alpha \mid \mathfrak{Z})$ is the spectrum of $a$; that is, $\sigma(\alpha \mid \mathfrak{Z})$ is the set of complex numbers $z$ for which $z-a$ is singular in $\mathfrak{A}$. In case there is no ambiguity involved we shall use the simpler notation $\sigma(a)$ for the spectrum of $a$.

If $X$ and $Y$ are Banach spaces, $\mathscr{L}(\mathfrak{X}, \mathfrak{Y})$ is the space of all continuous linear transformations from $\mathfrak{X}$ into $\mathfrak{Y}$, and $\mathscr{L}(\mathfrak{X})=\mathscr{L}(\mathfrak{X}, \mathfrak{X})$. If $\Omega$ is an index set we sometimes use $\bigcup\left\{a_{\lambda}: \lambda \in \Omega\right\}$ to mean $\bigcup_{\lambda \in \Omega} a_{\lambda}$.

1. Integral representation of inverses. Throughout this section $\left\{u_{j}\right\}_{j=0}^{n}$ and $\left\{v_{j}\right\}_{j=1}^{m}$ are subsets of $\mathfrak{i}$ that satisfy the following commutativity relations: $v_{j} v_{k}=v_{k} v_{j}$ and $v_{j} u_{\imath}=u_{i} v_{j}$ for $j, k=1, \cdots, m$ and $i=0, \cdots, n$. It should be noted that we do not require the $u_{j}$ to pairwise commute. $p_{0}, \cdots, p_{n}$ shall be polynomials in $m$ variables.

LEMMA 1.1. If

$$
\sigma\left(v_{1}\right) \times \cdots \times \sigma\left(v_{m}\right) \leqq\left\{\left(z_{1}, \cdots, z_{m}\right): \sum_{j=0}^{n} u_{j} p_{j}\left(z_{1}, \cdots, z_{m}\right) \text { is invertible }\right\} \text {, }
$$

then $\sum_{j=0}^{n} u_{j} p_{j}\left(v_{1}, \cdots, v_{m}\right)$ is invertible and its inverse is

$$
\left(\frac{1}{2 \pi i}\right)^{m} \int_{C_{1}} \cdots \int_{C_{m}}\left(\sum_{j=0}^{n} u_{j} p_{j}\left(z_{1}, \cdots, z_{m}\right)\right)^{-1} \prod_{k=1}^{m}\left(z_{k}-v_{k}\right)^{-1} d z_{1} \cdots d z_{m},
$$

where $C_{k}$ is the boundary of a Cauchy domain $D_{k}$ (see Taylor [13]) that contains $\sigma\left(v_{k}\right)$ for $k=1, \cdots, m$ and such that $\sum_{j=0}^{n} u_{j} p_{j}\left(z_{1}, \cdots\right.$, $\left.z_{m}\right)$ is invertible for $\left(z_{1}, \cdots, z_{m}\right)$ in $\bar{D}_{1} \times \cdots \times \bar{D}_{m}$.

Proof. The proof is by induction on $m$. 
Assume $m=1$. We shall show by direct computation that

$$
\begin{aligned}
\left(\sum_{j=0}^{n} u_{j} p_{j}\left(v_{1}\right)\right)^{-1}= & \frac{1}{2 \pi i} \int_{C_{1}}\left(\sum_{j=0}^{n} u_{j} p_{j}\left(z_{1}\right)\right)^{-1}\left(z_{1}-v_{1}\right)^{-1} d z_{1} \\
& \left(\sum_{k=0}^{n} u_{k} p_{k}\left(v_{1}\right)\right) \frac{1}{2 \pi i} \int_{C_{1}}\left(\sum_{j=0}^{n} u_{j} p_{j}\left(z_{1}\right)\right)^{-1}\left(z_{1}-v_{1}\right)^{-1} d z_{1} \\
= & \frac{1}{2 \pi i} \int_{C_{1}}\left(\sum_{k=0}^{n} u_{k}\left[p_{k}\left(v_{1}\right)^{-} p_{k}\left(z_{1}\right)\right]\right)\left(\sum_{j=0}^{n} u_{j} p_{j}\left(z_{1}\right)\right)^{-1}\left(z_{1}-v_{1}\right)^{-1} d z_{1} \\
& +\frac{1}{2 \pi i} \int_{C_{1}}\left(\sum_{k=0}^{n} u_{k} p_{k}\left(z_{1}\right)\right)\left(\sum_{j=0}^{n} u_{j} p_{j}\left(z_{1}\right)\right)^{-1}\left(z_{1}-v_{1}\right)^{-1} d z_{1} .
\end{aligned}
$$

Since $v_{1}-z_{1}$ and $\sum_{j=0}^{n} u_{j} p_{j}\left(z_{1}\right)$ commute, the penultimate term has an analytic integrand, and thus equals the zero element. The last term reduces to

$$
\frac{1}{2 \pi i} \int_{C_{1}}\left(z_{1}-v_{1}\right)^{-1} d z_{1}=1
$$

Thus the right term of (6) is a right inverse of $\sum_{j=0}^{n} u_{j} p_{j}\left(v_{1}\right)$. A similar computation shows that it is also a left inverse, which completes the proof for the case $m=1$.

Assume that the lemma is true when $m=k$, and that $\sigma\left(v_{1}\right) \times$ $\cdots \times \sigma\left(v_{k+1}\right) \subseteq\left\{\left(z_{1}, \cdots, z_{k+1}\right): \sum_{j=0}^{n} u_{j} p_{j}\left(z_{1}, \cdots, z_{k+1}\right)\right.$ is invertible $\}$.

Then for each $z_{k+1} \in \bar{D}_{k+1}$ the induction hypothesis yields

$$
\begin{aligned}
& \left(\sum_{j=0}^{n} u_{j} p_{j}\left(v_{1}, \cdots, v_{k}, z_{k+1}\right)\right)^{-1} \\
= & \left(\frac{1}{2 \pi i}\right)^{k} \int_{C_{1}} \cdots \int_{C_{k}}\left(\sum_{j=0}^{n} u_{j} p_{j}\left(z_{1}, \cdots, z_{k+1}\right)\right)^{-1} \prod_{k=1}^{n}\left(z_{k}-v_{k}\right)^{-1} d z_{1} \cdots d z_{k} .
\end{aligned}
$$

However, $\left(\sum_{j=0}^{n} u_{j} p_{j}\left(v_{1}, \cdots, v_{k}, z\right)\right)^{-1}$ is analytic for $z$ in a neighborhood of $\sigma\left(v_{k+1}\right)$. Thus, if we multiply (7) by $1 / 2 \pi i\left(z_{k+1}-v_{k+1}\right)^{-1}$, integrate about $C_{k+1}$, and apply (6), we deduce that the lemma is true for $m=k+1$.

We cite one special case of Lemma 1.1.

CoRollary 1.2. Suppose $\left\{u_{j}\right\}_{j=0}^{n}$ is a subset of the Banach algebra $\mathfrak{Z}$ and $v$ in $\mathfrak{X}$ commutes with $\left\{u_{j}\right\}_{j=0}^{n}$. If

$$
\sigma(v) \subseteq\left\{z: \sum_{j=0}^{n} u_{j} z^{j} \text { is invertible }\right\},
$$

then $\sum_{j=0}^{n} u_{j} v^{j}$ is invertible and

$$
\left(\sum_{j=0}^{n} u_{j} v^{j}\right)^{-1}=\frac{1}{2 \pi i} \int_{C}\left(\sum_{j=0}^{n} u_{j} z^{j}\right)^{-1}(z-v)^{-1} d z
$$

where $C$ is the boundary of a Cauchy domain $D$ that contains $\sigma(v)$ 
and such that $\sum_{j=0}^{n} u_{j} z^{j}$ is invertible for each $z$ in $\bar{D}$.

Lemma 1.1 enables us to infer the following general result about spectral inclusion as well as to write an explicit representation for the inverse of $\sum_{j=0}^{n} u_{j} p_{j}\left(v_{1}, \cdots, v_{m}\right)-\lambda$ for certain complex numbers $\lambda$.

\section{THEOREM 1.3.}

$$
\sigma\left(\sum_{j=0}^{n} u_{j} p_{j}\left(v_{1}, \cdots, v_{m}\right)\right) \subseteq \Delta
$$

where

$$
\Delta \stackrel{\text { def }}{=} \bigcup\left\{\sigma\left(\sum_{j=0}^{n} u_{j} p_{j}\left(z_{1}, \cdots, z_{m}\right)\right): z_{k} \in \sigma\left(v_{k}\right), k=1, \cdots, m\right\} .
$$

If $\lambda \notin \Delta$, then

$$
\begin{aligned}
\left(\sum_{j=0}^{n} u_{j} p_{j}\left(v_{1}, \cdots, v_{m}\right)-\lambda\right)^{-1} & \\
= & \left(\frac{1}{2 \pi i}\right)^{m} \int_{C_{1}} \cdots \int_{C_{m}}\left(\sum_{j=0}^{n} u_{j} p_{j}\left(z_{1}, \cdots, z_{m}\right)-\lambda\right)^{-1} \\
& \times \prod_{k=1}^{m}\left(z_{k}-v_{k}\right)^{-1} d z_{1} \cdots d z_{m},
\end{aligned}
$$

where $C_{k}$ is the boundary of a Cauchy domain $D_{k}$ that contains $\sigma\left(v_{k}\right)$ for $k=1, \cdots, m$ and such that $\sum_{j=0}^{n} u_{j} p_{j}\left(z_{1}, \cdots, z_{m}\right)-\lambda$ is invertible for $\left(z_{1}, \cdots, z_{m}\right) \in \bar{D}_{1} \times \cdots \times \bar{D}_{m}$.

Proof. If $\lambda \notin \Delta$, then it is immediate that $\sigma\left(v_{1}\right) \times \cdots \times \sigma\left(v_{m}\right) \subseteq$ $\left\{\left(z_{1}, \cdots, z_{m}\right): \sum_{j=0}^{n} u_{j} p_{j}\left(z_{1}, \cdots, z_{m}\right)-\lambda\right.$ is invertible $\}$. Define $u_{n+1}=-\lambda$ and $p_{n+1}=1$. Lemma 1.1 is now applicable to $\sum_{j=0}^{n+1} u_{j} p_{j}\left(v_{1}, \cdots, v_{m}\right)$. Thus the theorem follows from that lemma.

Simple finite dimensional examples show that the spectral containment conclusion of (8) need not hold, if the $v_{j}$ do not commute with $\left\{u_{k}\right\}$. Consider

$$
u_{0}=\left(\begin{array}{ll}
1 & 0 \\
0 & 0
\end{array}\right), u_{1}=\left(\begin{array}{ll}
0 & 1 \\
0 & 0
\end{array}\right) \text {, and } v_{1}=\left(\begin{array}{ll}
0 & 0 \\
1 & 0
\end{array}\right) .
$$

In this case $\sigma\left(u_{0}+u_{1} v_{1}\right)=\{0,2\}$, but $\bigcup\left\{\sigma\left(u_{0}+u_{1} z\right): z \in \sigma\left(v_{1}\right)\right\}=\{0,1\}$.

Even when the required commutativity relations hold one cannot in general hope for equality in (8). For example, consider commuting elements $u$ and $v$ of a Banach algebra, set $v_{1}=v_{2}=v$. Then $\sigma\left(u v_{1}-\right.$ $\left.u v_{2}\right)=\{0\}$, but $\bigcup\left\{\sigma\left(u z_{1}-u z_{2}\right): z_{1}, z_{2} \in \sigma(v)\right\}$ is in general not $\{0\}$.

2. A spectral mapping theorem. In $\S 1$ we showed that under 
certain commutativity conditions (8) holds, but that in general equality does not hold. In this section we find conditions sufficient to imply equality in (8). For a different attack, see Harte [5], [6].

Definition 2.1. Let $\mathfrak{X}$ be a complex Banach algebra with closed subalgebras $\mathfrak{N}_{0}, \mathfrak{N}_{1}, \cdots, \mathfrak{N}_{m}(m \geqq 1)$ such that the identity 1 in $\mathfrak{A}$ is also in $\mathfrak{A}_{j}, j=0, \cdots, m$. Then $\mathfrak{A}_{0}, \cdots, \mathfrak{N}_{m}$ are independent algebras in $\mathfrak{i}$ if the following conditions hold for $j, k=0, \cdots, m$ :

(i) If $a \in \mathfrak{A}_{j}, b \in \mathfrak{A}_{k}, j \neq k$, then $a b=b a$;

(ii) There exists a real number $M$ such that whenever $a_{j} \in \mathfrak{A}_{j}, j=0, \cdots, m$, then

$$
\prod_{j=0}^{m}\left\|a_{j}\right\| \leqq M\left\|a_{0} a_{1} \cdots a_{m}\right\|
$$

(iii) If $a_{j} \in \mathfrak{N}_{j}$, then $\sigma\left(a_{j} \mid \mathfrak{A}_{j}\right)=\sigma\left(a_{j} \mid \mathfrak{U}\right)$.

Lemma 2.2. Let $\mathfrak{N}_{0}, \cdots, \mathfrak{N}_{m}$ be independent algebras in $\mathfrak{A}$ with $\left\{u_{j}\right\}_{j=0}^{n} \subseteq \mathfrak{N}_{0}$ and $v_{k} \in \mathfrak{A}_{k}$ for $k=1, \cdots, m$. Let each of $p_{j}, j=0, \cdots$, $n$ be a polynomial in $m$ variables. If

$$
0 \in \bigcup\left\{\sigma\left(\sum_{j=0}^{n} u_{j} p_{j}\left(z_{1}, \cdots, z_{m}\right)\right): z_{k} \in \sigma\left(v_{k}\right), k=1, \cdots, m\right\},
$$

then there exist $\lambda_{k} \in \sigma\left(v_{k}\right), k=1, \cdots, m$ such that $\sum_{\jmath=0}^{n} u_{j} p_{j}\left(\lambda_{1}, \cdots, \lambda_{m}\right)$ is singular in $\mathfrak{U}$ and either

(i) $v_{k}-\lambda_{k}$ is the limit of invertible elements of $\mathfrak{N}_{k}$ for $k=1$, $\cdots, m$, or

(ii) $\sum_{j=0}^{n} u_{j} p_{j}\left(\lambda_{1}, \cdots, \lambda_{m}\right)$ is the limit of invertible elements of $\mathfrak{\imath}_{0}$.

Proof. Select a point $\left(\zeta_{1}, \cdots, \zeta_{m}\right)$ in $\sigma\left(v_{1}\right) \times \cdots \times \sigma\left(v_{m}\right)$ for which $\sum_{j=0}^{n} u_{j} p_{j}\left(\zeta_{1}, \cdots, \zeta_{m}\right)$ is singular. The select components $W_{k}$ of $\sigma\left(v_{k}\right)$ containing $\zeta_{k}$ for $k=1, \cdots, m$, and set $W=W_{1} \times \cdots \times W_{m}$. Clearly $W$ is a connected set in complex $m$-space. Let $K$ be the set of all points $\left(z_{1}, \cdots, z_{m}\right)$ in $W$ for which $\sum_{j=0}^{n} u_{j} p_{j}\left(z_{1}, \cdots, z_{m}\right)$ is invertible in $\mathfrak{2}_{0}$. Note that $K$ is open in $W$ and $K \neq W$. Thus since $W$ is connected, either $K$ is empty or there exists a point $\left(\lambda_{1}, \cdots, \lambda_{m}\right)$ in $\bar{K}-K$.

Case (a). If $K$ is empty, then $\sum_{j=0}^{n} u_{j} p_{j}\left(z_{1}, \cdots, z_{m}\right)$ is singular for all $\left(z_{1}, \cdots, z_{m}\right)$ in $W$. In particular it is singular for $\left(z_{1}, \cdots, z_{m}\right)$ chosen so that $z_{k}$ is in the boundary of $\sigma\left(v_{k}\right)=\sigma\left(v_{k} \mid \mathfrak{A}_{k}\right), k=1, \cdots, m$. Thus, (i) follows. See Rickart ([10], p. 22).

Case (b). Assume $\left(\lambda_{1}, \cdots, \lambda_{m}\right) \in \bar{K}-K$. This means that 


$$
\sum_{j=0}^{n} u_{j} p_{j}\left(\lambda_{1}, \cdots, \lambda_{m}\right)
$$

is singular, but is the limit of invertible elements of $\mathfrak{A}_{0}$. Thus (ii) holds.

We shall use the following terminology in the remeinder of this section. An element $u$ of $\mathfrak{A}_{j}$ is an $\mathfrak{A}_{j}$ generalized divisor of zero if there exists a sequence $\left\{x_{j}\right\}$ of unit vectors in $\mathfrak{A}_{j}$ such that $\lim _{j \rightarrow \infty} u x_{j}=$ 0 or $\lim _{j \rightarrow \infty} x_{j} u=0$. In the first case we say that $\left\{x_{j}\right\}$ right zero divides $u$ and in the second $\left\{x_{j}\right\}$ left zero divides $u$.

THEOREM 2.3. Assume that $\mathfrak{N}_{0}, \mathfrak{N}_{1}, \cdots, \mathfrak{N}_{m}$ are independent algebras in $\mathfrak{N}$ and that each singular element of $\mathfrak{N}_{j}$ is an $\mathfrak{N}_{j}$ generalized divisor of zero. If $\left\{u_{j}\right\}_{j=0}^{n} \subseteq \mathfrak{N}_{0,}, v_{k} \in \mathfrak{N}_{k}, k=1, \cdots, m$, and each of $p_{1}, \cdots, p_{m}$ is a polynomial, then

$$
\begin{aligned}
& \sigma\left(\sum_{j=0}^{n} u_{j} p_{j}\left(v_{1}, \cdots, v_{m}\right)\right) \\
& \quad=\bigcup\left\{\sigma\left(\sum_{j=0}^{n} u_{j} p_{j}\left(z_{1}, \cdots, z_{m}\right)\right): z_{k} \in \sigma\left(v_{k}\right), k=1, \cdots, m\right\} .
\end{aligned}
$$

Proof. Theorem 1.3 gives the containment $\subseteq$ in (10). To prove the reverse containment it is sufficient to assume that $0 \in \sigma\left(v_{k}\right), k=$ $1, \cdots, m, p_{j}(0, \cdots, 0)=0$ if $j \geqq 1$, and $0 \in \sigma\left(u_{0}\right)$, and deduce that $R=$ $\sum_{j=0}^{n} u_{j} p_{j}\left(v_{1}, \cdots, v_{m}\right)$ is not invertible. By hypothesis we know that there exist left or right zero-dividing sequences $\left\{y_{j}^{(0)}\right\} \cong \mathfrak{U}_{0}$ of $u_{0}$ and $\left\{y_{j}^{(k)}\right\} \subseteq \mathfrak{A}_{k}$ of $v_{k}, k=1, \cdots, m$. By Lemma 2.2 and the nature of limits of invertible operators (Rickart [10], p. 22) we may assume that either $y_{j}^{(0)}$ left divides $u_{0}$ or $\left\{y_{j}^{(k)}\right\}$ right divides $v_{k}$ for $k=1, \cdots$, $m$. Thus the following two cases exhaust all the possibilities.

Case (a). $\left\{y_{j}^{(0)}\right\}$ left divides $u_{0},\left\{y_{j}^{(k)}\right\}$ left divides $v_{k}$ for $k=1, \cdots$, $r$, and $y_{j}^{(k)}$ right divides $v_{k}$ for $k=r+1, \cdots, m$.

Assume that $R$ is invertible and set $g_{j}=R^{-1} y_{j}^{(r+1)} \cdots y_{j}^{(m)}$. Then if $k=r+1, \cdots, m, R\left(v_{k} g_{j}\right)=v_{k} R g_{j}=v_{k} y_{j}^{(r+1)} \cdots y_{j}^{(m)} \rightarrow 0$ as $j \rightarrow \infty$. Thus since $R$ is invertible $\lim _{j \rightarrow \infty} v_{k} g_{j}=0$ for $k=r+1, \cdots, m$. Then

$$
\begin{aligned}
& y_{j}^{(0)} \cdots y_{j}^{(m)}=y_{j}^{(0)} \cdots y_{j}^{(r)} R g_{j} \\
& \quad=y_{j}^{(0)} \cdots y_{j}^{(r)}\left[u_{0}+\sum_{j=1}^{n} u_{j} p_{j}\left(v_{1}, \cdots, v_{m}\right)\right] g_{j} \longrightarrow 0 \text { as } j \longrightarrow \infty .
\end{aligned}
$$

However, by condition (ii) of Definition 2.1,

$$
1=\prod_{k=0}^{m}\left\|y_{j}^{(k)}\right\| \leqq M\left\|y_{j}^{(0)} \cdots y_{j}^{(m)}\right\|,
$$

which is a contradiction, so $R$ cannot be invertible. 
For the remaining case $u_{0}$ does not have a left zero dividing sequence.

Case (b). $\quad\left\{y_{j}^{(k)}\right\}$ right divides $v_{k}$ for $k=1, \cdots, m$ and $\left\{y_{j}^{(0)}\right\}$ right divides $u_{0}$.

In this case

$$
\begin{aligned}
& R\left(y_{j}^{(0)} \cdots y_{j}^{(m)}\right)=u_{0} y_{j}^{(0)} \cdots y_{j}^{(m)}+\sum_{j=1}^{n} u_{j} y_{j}^{(0)} p_{j}\left(v_{1}, \cdots, v_{m}\right) y_{j}^{(1)} \cdots y_{j}^{(m)} \\
& \longrightarrow 0 \text { as } j \longrightarrow \infty .
\end{aligned}
$$

This shows that $R$ is not invertible since, as shown in the proof of case (a), $y_{j}^{(0)} \cdots y_{j}^{(m)}$ is bounded away from 0 .

We note that the "uniform crossnorm" condition (ii) of Definition 2.1 cannot be omitted in the hypotheses of Theorem 2.3. For, consider a Hilbert space $\mathfrak{S}_{1}$ and let $\mathfrak{S}_{\mathfrak{Z}}=\mathfrak{S}_{1} \oplus \mathfrak{S}_{1}$. Let $\mathfrak{H}_{0}$ and $\mathfrak{S}_{1}$ be defined by

$$
\begin{aligned}
& \mathfrak{U}_{0}=\left\{\left(\begin{array}{ll}
A & 0 \\
0 & \alpha I
\end{array}\right): A \in \mathscr{L}\left(\mathfrak{F}_{1}\right), \alpha \text { complex }\right\}, \\
& \mathfrak{N}_{1}=\left\{\left(\begin{array}{ll}
\beta I & 0 \\
0 & B
\end{array}\right): B \in \mathscr{L}\left(\mathscr{S}_{\mathcal{C}_{1}}\right), \beta \text { complex }\right\},
\end{aligned}
$$

and let $\mathfrak{Z}=\mathscr{L}(\mathfrak{S})$. Clearly $\mathfrak{S}_{\mathcal{E}}, \mathfrak{F}_{0}$, and $\mathfrak{U}_{1}$ satisfy all of the conditions of Definition 2.1 except possibly (ii). If we let $u_{0}=\left(\begin{array}{ll}A & 0 \\ 0 & 0\end{array}\right)$ and $v_{1}=$ $\left(\begin{array}{ll}0 & 0 \\ 0 & B\end{array}\right), A, B \in \mathscr{L}\left(\mathfrak{S}_{1}\right)$, then $\sigma\left(u_{0} v_{1}\right)=\{0\}$, but $\bigcup\left\{\sigma\left(u_{0} z\right): z \in \sigma\left(v_{1}\right)\right\}=$ $\sigma(A) \cdot \sigma(B) \cup\{0\}$. Thus in general $\sigma\left(u_{0} v_{1}\right) \neq \sigma\left(u_{0}\right) \sigma\left(v_{1}\right)$, and for this simple example the conclusion of Theorem 2.3 does not hold.

3. Applications. Our first two applications of Theorems 1.3 and 2.3 generalize results of Rosenblum [11, Theorem 3.1] and Lumer and Rosenblum [8, Theorem 10].

THEOREM 3.1. Suppose that $\left\{u_{j}\right\}_{j=0}^{n} \subseteq \mathfrak{A}$ and suppose $\left\{v_{k}\right\}_{k=1}^{m}$ is a commutative subset of $\mathfrak{X}$. Let each of $p_{j}, j=0, \cdots, n$, be a polynomial in $m$ variables. Define $R: \mathfrak{i} \rightarrow \mathfrak{U}$ by $R x=\sum_{j=0}^{n} u_{j} x p_{j}\left(v_{1}, \cdots, v_{m}\right)$. Then $\sigma(R) \subseteq \Delta$, where

$$
\Delta \stackrel{\text { ief }}{=} \bigcup\left\{\sigma\left(\sum_{j=0}^{n} u_{j} p_{j}\left(z_{1}, \cdots, z_{m}\right)\right): z_{k} \in \sigma\left(v_{k}\right), k=1, \cdots, m\right\},
$$

and if $\lambda \notin \Delta, x \in A$,

$$
\begin{aligned}
(R-\lambda)^{-1} x= & \left(\frac{1}{2 \pi i}\right)^{m} \int_{C_{1}} \cdots \int_{C_{m}}\left(\sum_{j=0}^{n} u_{j} p_{j}\left(z_{1}, \cdots, z_{m}\right)-\lambda\right)^{-1} \\
& \times x \prod_{k=1}^{m}\left(z_{k}-v_{k}\right)^{-1} d z_{1} \cdots d z_{m}
\end{aligned}
$$


where the $C_{k}$ are chosen as in Theorem 1.3.

Proof. Let $\mathfrak{B}=\mathscr{L}(\mathfrak{U})$,

$$
\begin{aligned}
& \mathfrak{B}_{0}=\left\{u^{L}: u \in \mathfrak{X} \text { and } u^{L}: x \longrightarrow u x, x \in \mathfrak{X}\right\} \\
& \mathfrak{B}_{1}=\left\{v^{R}: v \in \mathfrak{X} \text { and } v^{R}: x \longrightarrow x v, x \in \mathfrak{X}\right\} .
\end{aligned}
$$

By hypothesis $\mathfrak{B}_{1}$ is commutative, and clearly each element of $\mathfrak{B}_{0}$ commutes with each element of $\mathfrak{B}_{1}$. Thus we may apply Theorem 1.3 to $\sum_{j=0}^{n} u_{j}^{L} p_{j}\left(v_{1}^{R}, \cdots, v_{m}^{R}\right)$. Since $\sigma(R)=\sigma\left(\sum_{j=0}^{n} u_{j}^{L} p_{j}\left(v_{1}^{R}, \cdots, v_{m}^{R}\right)\right)$ and $\sigma\left(v_{k}\right)=\sigma\left(v_{k}^{R}\right)$, we have the desired conclusions.

A result analogous to that in Theorem 3.1 can be obtained if one fixes complex Banach spaces $\mathfrak{X}_{0}$ and $\mathfrak{X}_{1}$ and defines $R$ on $\mathscr{L}\left(\mathfrak{X}_{1}, \mathfrak{X}_{0}\right)$ by $R x=\sum_{j=0}^{n} u_{j} x p_{j}\left(v_{1}, \cdots, v_{m}\right)$, where $\left\{u_{j}\right\}_{j=0}^{n}$ is a subset of $\mathscr{L}\left(\mathfrak{X}_{0}\right)$ and $\left\{v_{k}\right\}_{k=1}^{m}$ is a commutative subset of $\mathscr{L}\left(\mathfrak{X}_{1}\right)$. Indeed, if we consider the case where $m=1$ we get a stronger result.

THeOREm 3.2. Let $\mathfrak{X}_{0}$ and $\mathfrak{X}_{1}$ be complex Banach spaces, $\left\{u_{j}\right\}_{j=0}^{n} \subseteq$ $\mathscr{L}\left(\mathfrak{X}_{0}\right)$ and $v \in \mathscr{L}\left(\mathfrak{X}_{1}\right)$. Define $R: \mathscr{L}\left(\mathfrak{X}_{1}, \mathfrak{X}_{0}\right) \rightarrow \mathscr{L}\left(\mathfrak{X}_{1}, \mathfrak{X}_{0}\right)$ by

$$
R x=\sum_{j=0}^{n} u_{j} x v^{j} .
$$

Then

$$
\sigma(R)=\bigcup\left\{\sigma\left(\sum_{j=0}^{n} u_{j} z^{j}\right): z \in \sigma(v)\right\}
$$

and if $\lambda \notin \sigma(R)$,

$$
(R-\lambda)^{-1} x=\frac{1}{2 \pi i} \int_{C}\left(\sum_{j=0}^{n} u_{j} z^{j}-\lambda\right)^{-1} x(z-v)^{-1} d z,
$$

where $C$ is the boundary of a Cauchy domain $D$ that contains $\sigma(v)$ and such that $\sum_{j=0}^{n} u_{j} z^{j}$ is invertible for $z$ in $\bar{D}$.

$$
\begin{aligned}
& \text { Proof. Let } \mathfrak{A}=\mathscr{L}\left(\mathscr{L}\left(\mathfrak{X}_{1}, \mathfrak{X}_{0}\right)\right), \\
& \mathfrak{N}_{0}=\left\{u^{L}: u \in \mathscr{L}\left(\mathfrak{X}_{0}\right) \text { and } u^{L}: x \longrightarrow u x, x \in \mathscr{L}\left(\mathfrak{X}_{1}, \mathfrak{X}_{0}\right)\right\},
\end{aligned}
$$

and

$$
\mathfrak{U}_{0}=\left\{v^{R}: v \in \mathscr{L}\left(\mathfrak{X}_{1}\right) \text { and } v^{R}: x \longrightarrow x v, x \in \mathscr{L}\left(\mathfrak{X}_{1}, \mathfrak{X}_{0}\right)\right\} .
$$

It is easily checked that conditions (i) and (iii) of Definition 2.1 are satisfied by $\mathfrak{A}, \mathfrak{N}_{0}$, and $\mathfrak{N}_{1}$. The following argument will show that the "uniform crossnorm" condition (ii) is also satisfied and thus by 
Theorem 2.3

$$
\sigma(R)=\sigma\left(\sum_{j=0}^{n} u_{j}^{L}\left(v^{R}\right)^{j}\right)=\bigcup\left\{\sigma\left(\sum_{j=0}^{n} u_{j}^{L} z^{j}\right): z \in \sigma\left(v^{R}\right)\right\} .
$$

This is the desired conclusion since $\sigma\left(v^{R}\right)=\sigma(v)$ and $\sigma\left(\sum_{j=0}^{n} u_{j}^{L} z^{j}\right)=$ $\sigma\left(\sum_{j=0}^{n} u_{j} z^{j}\right)$.

Choose unit vectors $\left\{\alpha_{n}\right\}$ in $X_{0}$ and $\left\{\beta_{n}\right\}$ in $\mathfrak{X}_{1}^{*}$ so that $\left\|u \alpha_{n}\right\| \rightarrow$ $\|u\|$ and $\left\|v^{*} \beta_{n}\right\| \rightarrow\|v\|$. Then, upon setting $x_{n}=\left\langle\cdot, \beta_{n}\right\rangle \alpha_{n}$, we have

$$
\begin{aligned}
\left\|u^{L} v^{R}\right\| & =\sup \left\{\|u x v\|: x \in \mathscr{L}\left(\mathfrak{X}_{1}, \mathfrak{X}_{0}\right),\|x\|=1\right\} \\
& \geqq \lim _{n} \sup \left\|u x_{n} v\right\| \\
& =\lim _{n} \sup \left(\left\|v^{*} \beta_{n}\right\|\left\|u \alpha_{n}\right\|\right)=\|u\|\|v\| .
\end{aligned}
$$

Consequently we have $\left\|u^{L} v^{R}\right\| \geqq\left\|u^{L}\right\|\left\|v^{R}\right\|$, which proves that Theorem 2.3 is applicable.

(11) was proved by Harte ([6], Theorem 3.5) under the assumption that $\mathfrak{X}_{0}=\mathfrak{X}_{1}$ is a Hilbert space.

Next we give an application of Theorem 2.3 similar to the one above to obtain a generalization of a result of Brown and Pearcy [1].

THEOREM 3.3. Let $\mathfrak{S}$ be a complex Hilbert space and let $c_{p}$ be the class of compact operators $u$ in $\mathscr{L}(\mathfrak{S})$ for which

$$
\|u\|_{p}=\left[\operatorname{tr}\left(u^{*} u\right)^{p / 2}\right]^{1 / p}<\infty \quad \text { if } \quad 1 \leqq p<\infty
$$

and $\|u\|_{\infty}=\|u\|$. Fix $u_{0}, \cdots, u_{n}, v$ in $\mathscr{L}(\mathfrak{S})$ and define $R: \mathscr{L}\left(c_{p}\right) \rightarrow$ $\mathscr{L}\left(c_{p}\right), 1 \leqq p \leqq \infty$ by $R x=\sum_{j=0}^{n} u_{j} x v^{j}$. Then (11) holds and if $\lambda \notin \sigma(R)$, so does (12).

Indication of proof. Let $\mathfrak{X}=\mathscr{L}\left(c_{p}\right)$ and proceed as in the proof of Theorem 3.2.

The remaining applications deal with tensor products. The authors were led to the formulation of Definition 2.1 and Theorem 2.3 through efforts to unify these results and the preceding applications. In the next theorem (13) can be deduced from Harte ([6], Theorem 2.3), and (14) is new.

THeOREM 3.4. Let $\mathfrak{X}_{0}, \mathfrak{X}_{1}, \cdots, \mathfrak{X}_{m}$ be complex Banach spaces and let $\mathfrak{X}$ be the completion of $\mathfrak{X}_{0} \otimes \mathfrak{X}_{1} \otimes \cdots \otimes \mathfrak{X}_{m}$ with respect to some uniform crossnorm. Let $\left\{u_{j}\right\}_{j=0}^{n} \subseteq \mathscr{L}\left(\mathfrak{X}_{0}\right), v_{k} \in \mathscr{L}\left(\mathfrak{X}_{k}\right), k=1, \cdots, m$ and let each of $p_{0}, \cdots, p_{n}$ be a polynomial in $m$ variables.

Define 


$$
\begin{aligned}
& u_{j}^{(0)}=u_{j} \otimes \overbrace{I \otimes \cdots \otimes I}^{m \text { terms }}, j=0, \cdots, n \\
& v^{(1)}=I \otimes v_{1} \otimes \cdots \otimes I \\
& \vdots \\
& v^{(m)}=I \otimes \cdots \otimes I \otimes v_{m} .
\end{aligned}
$$

Then

$$
\begin{aligned}
& \sigma\left(\sum_{j=0}^{n} u_{j}^{(0)} p_{j}\left(v^{(1)}, \cdots, v^{(m)}\right) \mid \mathscr{L}(\mathfrak{X})\right) \\
& =\bigcup\left\{\sigma\left(\sum_{j=0}^{n} u_{j} p_{j}\left(z_{1}, \cdots, z_{m}\right) \mid \mathscr{L}\left(\mathfrak{X}_{0}\right)\right): z_{k} \in \sigma\left(v_{k} \mid \mathscr{L}\left(\mathfrak{X}_{k}\right)\right),\right. \\
& k=1, \cdots, m\} \text {. }
\end{aligned}
$$

Moreover, if $\lambda \notin \sigma\left(\sum_{j=0}^{n} u_{j}^{(0)} p_{j}\left(v^{(1)}, \cdots, v^{(m)}\right) \mid \mathscr{L}\left(\mathfrak{X}_{0}\right)\right)$, then

$$
\begin{aligned}
\left(\sum_{j=0}^{n} u_{j}^{(0)} p_{j}\left(v^{(1)}, \cdots, v^{(m)}\right)-\lambda\right)^{-1} & \\
= & \left(\frac{1}{2 \pi i}\right)^{m} \int_{C_{1}} \cdots \int_{c_{m}}\left(\sum_{j=0}^{n} u_{j} p_{j}\left(z_{1}, \cdots, z_{m}\right)-\lambda\right)^{-1} \\
& \otimes\left(z_{1}-v_{1}\right)^{-1} \otimes \cdots \otimes\left(z_{m}-v_{m}\right)^{-1} d z_{1} \cdots d z_{m}
\end{aligned}
$$

where $C_{1}, \cdots, C_{m}$ is in Theorem 1.3 .

$$
\begin{aligned}
\text { Proof. Let } \mathfrak{N} & =\mathscr{L}(\mathfrak{X}) \text { and } \\
\mathfrak{N}_{0} & =\left\{u \otimes I \otimes \cdots \otimes I: u \in \mathscr{L}\left(\mathfrak{X}_{0}\right)\right\} \\
\mathfrak{R}_{1} & =\left\{I \otimes v_{1} \otimes \cdots \otimes I: v_{1} \in \mathscr{L}\left(\mathfrak{X}_{1}\right)\right\} \\
\vdots & \\
\mathfrak{N}_{m} & =\left\{I \otimes \cdots \otimes I \otimes v_{m}: v_{m} \in \mathscr{L}\left(\mathfrak{X}_{m}\right)\right\} .
\end{aligned}
$$

Each of $\mathfrak{A}_{0}, \cdots, \mathfrak{A}_{m}$ is a closed subalgebra of $\mathfrak{A}$ containing the identity $I \otimes \cdots \otimes I$. Since the crossnorm is uniform,

$$
\left\|a_{0} \cdots a_{m}\right\|=\left\|a_{0}\right\| \cdots\left\|a_{m}\right\| \text { for } a_{j} \in \mathfrak{R}_{j}, j=0, \cdots, m
$$

and thus it is easily seen that $\mathfrak{A}_{0}, \cdots, \mathfrak{A}_{m}$ are independent algebras in $\mathfrak{A}$. Each singular element of $\mathfrak{A}_{j}$ is an $\mathfrak{N}_{j}$ generalized zero divisor (Rickart [10], p. 279). Then by Theorem 2.3

$$
\begin{aligned}
& \sigma\left(\sum_{j=0}^{n} u_{j}^{(0)} p_{j}\left(v^{(1)}, \cdots, v^{(m)}\right) \mid \mathscr{L}(\mathfrak{X})\right) \\
& =\bigcup\left\{\sigma\left(\sum_{j=0}^{n} u_{j}^{(0)} p_{j}\left(z_{1}, \cdots, z_{m}\right) \mid \mathscr{L}(\mathfrak{X})\right): z_{k} \in \sigma\left(v^{(k)} \mid \mathscr{L}(\mathfrak{X})\right),\right. \\
& \quad k=1, \cdots, m\} .
\end{aligned}
$$


The result now follows since $\sigma\left(v^{(k)} \mid \mathscr{L}(\mathfrak{X})\right)=\sigma\left(v_{k} \mid \mathscr{L}\left(\mathfrak{X}_{k}\right)\right), k=1, \cdots$, $m$ and $\sigma\left(u^{(0)} \mid \mathfrak{A}\right)=\sigma\left(u \mid \mathfrak{A}_{0}\right)$ for any $u^{(0)}$ in $\mathfrak{A}$ of the form

$$
u^{(0)}=u \otimes I \otimes \cdots \otimes I, u \in \mathscr{L}\left(\mathfrak{X}_{0}\right) .
$$

As in the proofs of the preceding applications the representation formula (14) is a consequence of Theorem 1.3.

If in Theorem 3.4 we choose $u_{0}=v_{0} \in \mathscr{L}\left(\mathfrak{X}_{0}\right)$ and $u_{j}=0$ for $j=$ $1, \cdots, n$, we obtain Schechter's result [12, Theorem 2.1]:

$$
\begin{aligned}
& \sigma\left(p\left(v^{(0)}, v^{(1)}, \cdots, v^{(m)}\right)\right)=\bigcup\left\{\sigma\left(p\left(v_{0}, z_{1}, \cdots, z_{m}\right) \mid \mathscr{L}\left(\mathfrak{X}_{0}\right)\right):\right. \\
& \left.z_{j} \in \sigma\left(v_{j} \mid \mathscr{L}\left(\mathfrak{X}_{j}\right)\right), j=1, \cdots, m\right\}=p\left(\sigma\left(v_{0}\right), \sigma\left(v_{1}\right), \cdots, \sigma\left(v_{m}\right)\right)
\end{aligned}
$$

for any polynomial $p$ of $m+1$ variables. More specifically we have the following result.

CoRollary 3.5. Let $\mathfrak{X}_{1}, \cdots, \mathfrak{X}_{m}$ satisfy the hypotheses of Theorem 3.4 and let $v_{j} \in \mathscr{L}\left(\mathfrak{X}_{j}\right), j=1, \cdots, m$. Then

$$
\sigma\left(v_{1} \otimes \cdots \otimes v_{m}\right)=\prod_{k=1}^{m} \sigma\left(v_{k} \mid \mathscr{L}\left(\mathfrak{X}_{k}\right)\right),
$$

and if $\lambda \notin \sigma\left(v_{1} \otimes \cdots \otimes v_{m}\right)$, then

$$
\begin{gathered}
\left.\left(v_{1} \otimes \cdots \otimes v_{m}-\lambda\right)^{-1}=\left(\frac{1}{2 \pi i}\right)^{m} \int_{C_{1}} \cdots \int_{C_{m}}\left(z_{1} \cdots z_{m}-\lambda\right)^{-1}\right) \\
\otimes\left(z_{1}-v_{0}\right)^{-1} \otimes \cdots \otimes\left(z_{m}-v_{m}\right)^{-1} d z_{1} \cdots d z_{m}
\end{gathered}
$$

where $C_{1}, \cdots, C_{m}$ is as in Theorem 1.3.

Proof. Let $\mathfrak{X}_{0}$ be a one dimensional Hilbert space and set $u_{j}=$ $0, j \geqq 1, u_{0}=1, p_{0}\left(z_{1}, \cdots, z_{m}\right)=z_{1} \cdots z_{m}$ in Theorem 3.4.

Next we consider a complex Hilbert space $\sqrt{5}$ and let $H_{\varpi}^{2}\left(U^{m}\right)$ be the Hardy space of $\mathscr{L}(\sqrt{5})$-valued functions holomorphic in $U^{m}=U \times$ $\cdots \times U$ ( $m$ factors), where $U$ is the unit disk in the complex plane.

COROLLARY 3.6. Let $n$ be a nonnegative integer, and assume $\left\{c_{j_{1}, \cdots, j_{m}}: 0 \leqq j_{1}, \cdots, j_{m}<\infty\right\} \leqq \mathscr{L}(\mathfrak{S})$, where all but a finite numbers

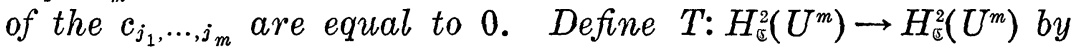

$$
T: f\left(z_{1}, \cdots, z_{m}\right) \longrightarrow \sum_{j_{1}}, \cdots, j_{m} c_{j_{1}, \cdots, j_{m}} z_{1}^{j_{1}} \cdots z_{m}^{j_{m}} f\left(z_{1}, \cdots, z_{m}\right) .
$$

Then

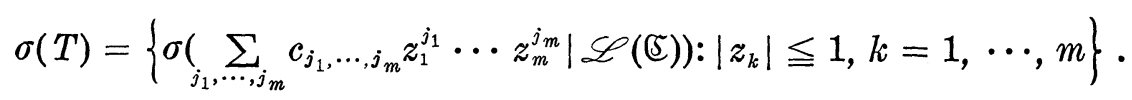


Proof. $H_{\varpi}^{2}\left(U^{m}\right)$ is the completion of $\sqrt{5} \otimes H^{2}(U) \otimes \cdots \otimes H^{2}(U)$ under the Hilbert tensor product norm. If $S$ is the unilateral shift on $H^{2}(U)$ defined by $(S f)(z)=z f(z)$, then one can view $T$ as

$$
T=\sum c_{j_{1}, \cdots, j_{m}} S^{j_{1}} \otimes \cdots \otimes S^{j_{m}} .
$$

The corollary now follows by applying (13) of Theorem 3.4 and noting that $\sigma(S)=\{z:|z| \leqq 1\}$.

Theorem 3.4 also leads to the following result:

COROLLARY 3.7. Let (5 be a complex Hilbert space, $\left\{u_{j}\right\}_{j=0}^{n} \subseteq$ $\mathscr{L}(\mathfrak{C})$ and define $V$ on $\sum_{j=0}^{\infty} \oplus \mathbb{C}$ by

$$
V:\left\{c_{j}\right\}_{j=0}^{\infty} \longrightarrow\left\{\sum_{k=0}^{n} u_{k} c_{k+j}\right\}_{j=0}^{\infty},
$$

so

$$
V=\left(\begin{array}{cccccccc}
u_{0} & u_{1} & \cdots & & u_{n} & 0 & 0 & \cdots \\
0 & u_{0} & u_{1} & \cdots & & u_{n} & 0 & \cdots \\
0 & 0 & u_{0} & u_{1} & \cdots & & u_{n} & \cdots \\
\vdots & \vdots & \ddots & & & & \ddots &
\end{array}\right)
$$

Then $\sigma(v)=\bigcup\left\{\sigma\left(\sum_{j=0}^{n} u_{j} z^{j}\right):|z| \leqq 1\right\}$.

Proof. $\sum_{j=0}^{\infty} \oplus \mathbb{C}$ is isomorphic to the Hilbert space $\mathbb{E} \otimes H^{2}(U)$ under the isomorphism that sends $\left\{c_{j}\right\}_{j=0}^{\infty}$ into $\sum_{j=0}^{\infty} c_{j} \otimes z^{j}$. If $S$ is the unilateral shift on $H^{2}(U), S f(z)=z f(z)$ then $V$ is mapped into

$$
\sum_{j=0}^{n} u_{j} \otimes S^{* j}
$$

Thus the corollary follows from Theorem 3.4. (14) can be used to set down a formula of $(v-\lambda)^{-1}$ if $\lambda \notin \sigma(v)$.

\section{REFERENCES}

1. A. Brown and C. Pearcy, Spectra of tensor products of operators, Proc. Amer. Math. Soc., 17 (1966), 162-169.

2. A. T. Dash and M. Schechter, Tensor products and joint spectra, Israel J. Math., 8 (1970), 191-193.

3. G. S. Datuasvili, The spectrum of a generalized matrix polynomial (in Russian, with Georgian summary), Sakharth SSR Mecn. Akad. Moambi, 44 (1966), 7-9.

4. J. Dixmier, Les algèbres d'opèrateurs dans l'espace Hilbertien, Gauthier-Villars, Paris, 1957.

5. R. E. Harte, Spectral mápping theorems, Proc. Royal Irish Acad., 72, Section A, number 7 (1972), 89-107. 
6. R. E. Harte, Tensor products, multiplication operators and the spectral mapping theorem, (preprint).

7. Peter Lancaster, Explicit solutions of linear matrix equations, SIAM Review, 12 (1970), 544-566.

8. G. Lumer and M. Rosenblum, Linear operator equations, Proc. Amer. Math. Soc., 10 (1959), 32-41.

9. C. C. MacDuffee, The theory of matrices, Ergebnisse der Math., 1933.

10. C. E. Rickart, General Theory of Banach Algebras, Van Nostrand, Princeton, 1960.

11. M. Rosenblum, On the operator equation $B X-X A=Q$, Duke Math. J., 23 (1956), 263-269.

12. M. Schechter, On the spectra of operators on tensor products, J. Functional Analysis, 4 (1969), 95-99.

13. A. Taylor, Introduction to Functional Analysis, Wiley, New York, 1958.

14. F. Treves, Topological Vector Spaces, Distributions and Kernels, Academic Press, New York, 1967.

Received July 24, 1973. M. Rosenblum was supported in part by NSF Grant GP-31483X.

University of North Carolina at Charlotte

AND

UNIVERSITY OF VIRGINIA 


\section{PACIFIC JOURNAL OF MATHEMATICS}

\section{EDITORS}

RICHARD ARENS (Managing Editor)

University of California

Los Angeles, California 90024

R. A. Beaumont

University of Washington

Seattle, Washington 98105
J. DugundJI

Department of Mathematics

University of Southern California

Los Angeles, California 90007

D. Gilbarg and J. Milgram

Stanford University

Stanford, California 94305

\section{ASSOCIATE EDITORS}

E. F. BECKENBACH

B. H. NeUMANN

F. WOLF

K. YosHIDA

\section{SUPPORTING INSTITUTIONS}

UNIVERSITY OF BRITISH COLUMBIA

CALIFORNIA INSTITUTE OF TECHNOLOGY

UNIVERSITY OF CALIFORNIA

MONTANA STATE UNIVERSITY

UNIVERSITY OF NEVADA

NEW MEXICO STATE UNIVERSITY

OREGON STATE UNIVERSITY

UNIVERSITY OF OREGON

OSAKA UNIVERSITY
UNIVERSITY OF SOUTHERN CALIFORNIA

STANFORD UNIVERSITY

UNIVERSITY OF TOKYO

UNIVERSITY OF UTAH

WASHINGTON STATE UNIVERSITY

UNIVERSITY OF WASHINGTON

$\stackrel{*}{*} \stackrel{*}{*} \stackrel{*}{*}$ AMERICAN MATHEMATICAL SOCIETY




\section{Pacific Journal of Mathematics}

\section{Vol. 53, No. $1 \quad$ March, 1974}

Martin Bartelt, Strongly unique best approximates to a function on a set, and a finite

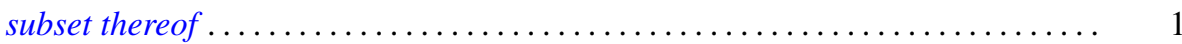

S. J. Bernau, Theorems of Korovkin type for $L_{p}$-spaces $\ldots \ldots \ldots \ldots \ldots \ldots \ldots \ldots \ldots$

S. J. Bernau and Howard E. Lacey, The range of a contractive projection on an

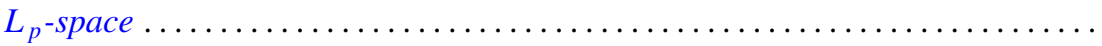

Marilyn Breen, Decomposition theorems for 3-convex subsets of the plane ......... Ronald Elroy Bruck, Jr., A common fixed point theorem for a commuting family of

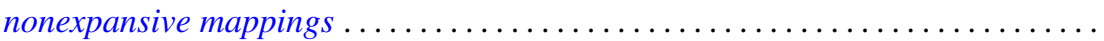

Aiden A. Bruen and J. C. Fisher, Blocking sets and complete $k$-arcs . . . . . . . 73

R. Creighton Buck, Approximation properties of vector valued functions . ......... 85

Mary Rodriguez Embry and Marvin Rosenblum, Spectra, tensor products, and

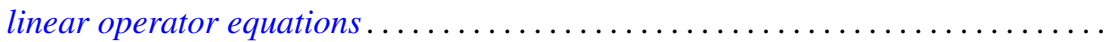

Edward William Formanek, Maximal quotient rings of group rings . . . . . . . . . 109

Barry J. Gardner, Some aspects of T-nilpotence . . . . . . . . . . . . . . . 117

Juan A. Gatica and William A. Kirk, A fixed point theorem for $k$-set-contractions

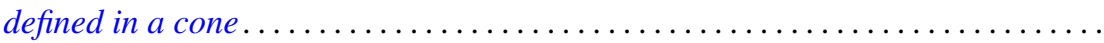

Kenneth R. Goodearl, Localization and splitting in hereditary noetherian prime

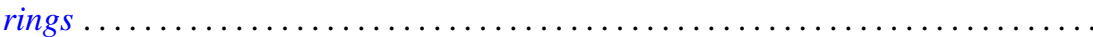

James Victor Herod, Generators for evolution systems with quasi continuous

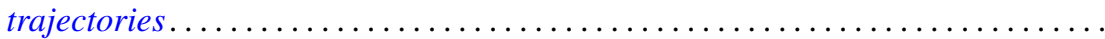

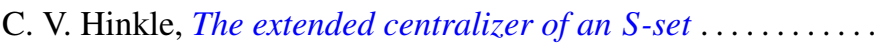

I. Martin (Irving) Isaacs, Lifting Brauer characters of p-solvable groups . . .

Bruce R. Johnson, Generalized Lerch zeta function ...........

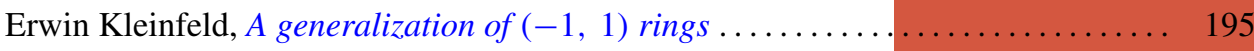

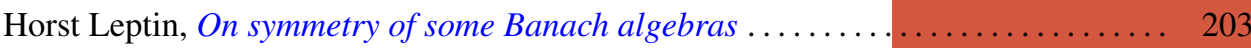

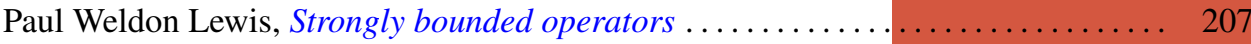

Arthur Larry Lieberman, Spectral distribution of the sum of self-adjoint

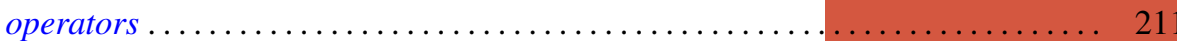

I. J. Maddox and Michael A. L. Willey, Continuous operators on paranormed spaces and matrix transformations

James Dolan Reid, On rings on groups ........................... 229

Richard Miles Schori and James Edward West, Hyperspaces of graphs are Hilbert cubes.

William H. Specht, A factorization theorem for p-constrained groups ...

Robert L Thele, Iterative techniques for approximation of fixed points of certain nonlinear mappings in Banach spaces ...............

Tim Eden Traynor, An elementary proof of the lifting theorem

Charles Irvin Vinsonhaler and William Jennings Wickless, Completely decomposable groups which admit only nilpotent multiplications .

Raymond O’Neil Wells, Jr, Comparison of de Rham and Dolbeault cohomology for

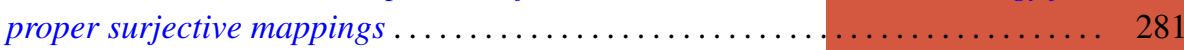

David Lee Wright, The non-minimality of induced central representations . . . . . 301 